\title{
Recent experiments on three nucleon systems and problems to be solved
}

\author{
Kenshi Sagara ${ }^{1, a}$ \\ Department of Physics, Kyushu University, Fukuoka, 812-8581, JAPAN
}

\begin{abstract}
After $2 \pi 3 N \mathrm{~F}$ was found in 1998 , many experiments were made on $N d$ elastic scattering, $N d$ breakup and $p d$ capture, and many discrepancies between experiments and calculations were revealed. Systematic experimental data are still being accumulated. From the systematic data, $3 N \mathrm{~F}$ other than $2 \pi 3 N \mathrm{~F}$ such as $\pi \rho 3 N \mathrm{~F}$ and $\rho \rho 3 N \mathrm{~F}$, and origins of low-energy anomalies are expected to be found in the future.
\end{abstract}

\section{Introduction}

One of the purposes to study three-nucleon $(3 N)$ systems is to find effects of three-nucleon forces $(3 N \mathrm{~F})$ and to determine their strengths. As is well known, Fujita-Miyazawa predicted existence of $2 \pi$-exchange three nucleon force $(2 \pi 3 N F)$ in $1957[1]$. Faddeev equations for $3 N$ systems have been numerically solved since late 1960's. In 1980's, it became widely known that ${ }^{3} \mathrm{H}$ binding energy cannot be reproduced by $2 N \mathrm{~F}$ alone and can be reproduced using $2 \pi 3 N \mathrm{~F}$ with an adjustable parameter. To justify the value of the parameter, further evidences were necessary.

From systematic measurement of $p d$ elastic scattering cross section in energy range of $E_{p}=2-18 \mathrm{MeV}$ at Kyushu University tandem laboratory (KUTL), systematic discrepancy between experiment and calculation in the cross section minima around $110^{\circ}$ was found in 1994[2]. The discrepancy was, however, paid no attention because Coulomb force was not correctly treated in $p d$ calculations at that time. In 1996, pd scattering cross section was measured at $E_{d}=270 \mathrm{MeV}\left(E_{p}=135 \mathrm{MeV}\right)$ to construct a $d$-beam polarimeter at RIKEN. Koike found by chance the same discrepancy at the cross section minimum also at 135 $\mathrm{MeV}$, and he introduced the discrepancy as Sagara discrepancy in FB15 in 1997[3]. In 1998, Witała et al., excellently solved the binding energy problem and Sagara discrepancy by introducing the same $2 \pi 3 N F[4]$.

After $2 \pi 3 N \mathrm{~F}$ was discovered, many theoretical studies and experiments on $3 N F$ have been made. The experimental studies have been widely made at higher energy region on $N d$ elastic scattering, and also made on $p d$ breakup and $p d$ capture.

In $p d$ elastic scattering, many kinds of spin observables, such as analyzing powers of $A_{y}, i T_{11}, A_{y y}, A_{x x}$ and $A_{x z}$, and polarization transfer coefficients have been measured. Also cross section of $p d$ elastic scattering was measured at various energies. In $p d$ breakup, cross section and $A_{y}$ have been measured. Our experiment on $p d$ breakup at $E_{p}=247 \mathrm{MeV}$ is presented in this conference[5]. In $p d$

\footnotetext{
a e-mail: sagara@phys.kyushu-u.ac.jp
}

capture, tensor analyzing powers $A_{y y}, A_{z z}$ and also $A_{x x}$ have been measured in the last decade at $E_{d}=100-200 \mathrm{MeV}$.

All the experimental observables at higher energy disagree more or less with calculations even after $2 \pi 3 N \mathrm{~F}$ being included. The disagreements seem, at least in part, to be caused by $3 N F$ other than $2 \pi 3 N F$. We report the disagreements in some detail later.

At low energy region, there are long-standing problems of $A_{y}$ puzzle and Space Star anomaly (SS anomaly), which seem to be irrelevant to $3 \mathrm{NF}$. Now, we have sufficient data for $A_{y}$ puzzle. Experimentalists are just waiting for theoretical investigations. As for SS anomaly, which is a discrepancy between experiment and calculation in $N d$ breakup cross section around $10 \mathrm{MeV}$, there were a few experiments in $20^{\text {th }}$ century, because there were no reliable calculations on $p d$ breakup and SS anomaly was studied only in $n d$ breakup. Experiments on $p d$ breakup are far more precise and far easier than $n d$ breakup experiments. Experimentalists desired for a long time for $p d$ breakup calculations. During the time, for example, combination of $n d$ breakup Faddeev calcualtion and Watson-Migdal $p p$ FSI formula was tried to approximate $p d$ breakup calculation, and experimental data were fairly well reproduced.

A breakthrough was made by A. Deltuva et al. in 2005[6]. They succeeded in calculations of all kinds of $p d$ reactions including $p d$ breakup using fast-damping screened Coulomb force. After the success of $p d$ breakup calculation, we started to measure $p d$ breakup cross section systematically, to search for origin(s) of the star anomaly.

Figure 1 illustrates discrepancies in $3 N$ systems. We have already solved discrepancies in $3 N$ binding energy and in $p d$ scattering cross section minimum by $2 \pi 3 N \mathrm{~F}$. There are still many disagreements remaining at higher energy as well as at lower energy. Some of the disagreements may indicate effects of $3 N F$ other than $2 \pi 3 N F$. 


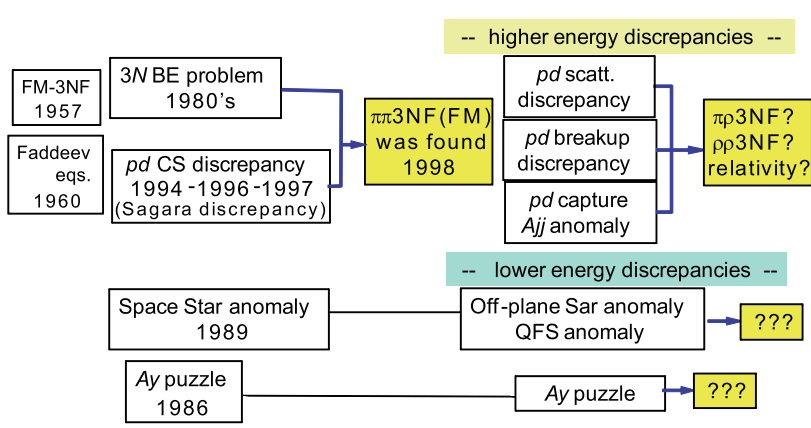

Fig. 1. Discrepancies between experiments and calculations in $3 N$ systems, in higher energy region and in lower energy region.

\section{Experiments on $p d$ elastic scattering at higher energy}

After $2 \pi 3 N \mathrm{~F}$ was discovered, many experiments were made on $p d$ elastic scattering and $2 \pi 3 N \mathrm{~F}$ effects were examined. As so many groups measured $p d$ and $n d$ elastic scattering, we Kyushu group did not measure it, instead, measured $p d$ breakup and $p d$ capture.

Below $E_{p}=200 \mathrm{MeV}$, the cross section minimum of elastic scattering was well reproduced by introducing $2 \pi 3 N \mathrm{~F}$. Above $140 \mathrm{MeV}$, it was found that the scattering cross section at backward angle becomes larger than calculation, and the disagreement increases monotonically with energy. Experimental values are about twice of calculated values at $250 \mathrm{MeV}$ [7]. This systematic disagreement seems to indicate effects of short-range $3 \mathrm{NF}$ other than $2 \pi 3 N \mathrm{~F}$ and/or relativistic effects.

Many kinds of polarization observables of $p d$ scattering have been measured. They were found to disagree with calculations. Disagreements in polarization observables are complicated and are not so large as that of the cross section (for example see [8]). Besides systematic feature like cross section enhancement has not been found in disagreements of polarization observables.

It may be better to investigate first the systematic disagreement in the cross section of elastic scattering, and to study other disagreements after the problems in the cross section are completely solved. Cross section is a basic scalar quantity, and modification of cross section influences more or less polarization observables.

\section{Experiments on $p d$ breakup at higher energy}

After $2 \pi 3 N \mathrm{~F}$ was discovered, we started $p d$ breakup experiment at $E_{p}=247 \mathrm{MeV}$ at RCNP. To see global feature, we first made $\mathrm{D}\left(p, p_{1}\right) p_{2} n$ experiment by detecting only one proton $p 1$ out of three outgoing nucleons. To significantly reduce backgrounds from the target, we used an almost pure liquid $\mathrm{D}_{2}$ target instead of an ordinary $\mathrm{CD}_{2}$ target. We had developed the liquid hydrogen target for our $p d$ capture experiment described below.

Experimental results for $\mathrm{D}\left(p, p_{1}\right) p_{2} n$ cross section at $247 \mathrm{MeV}$ are shown in Figure 2 with calculations by

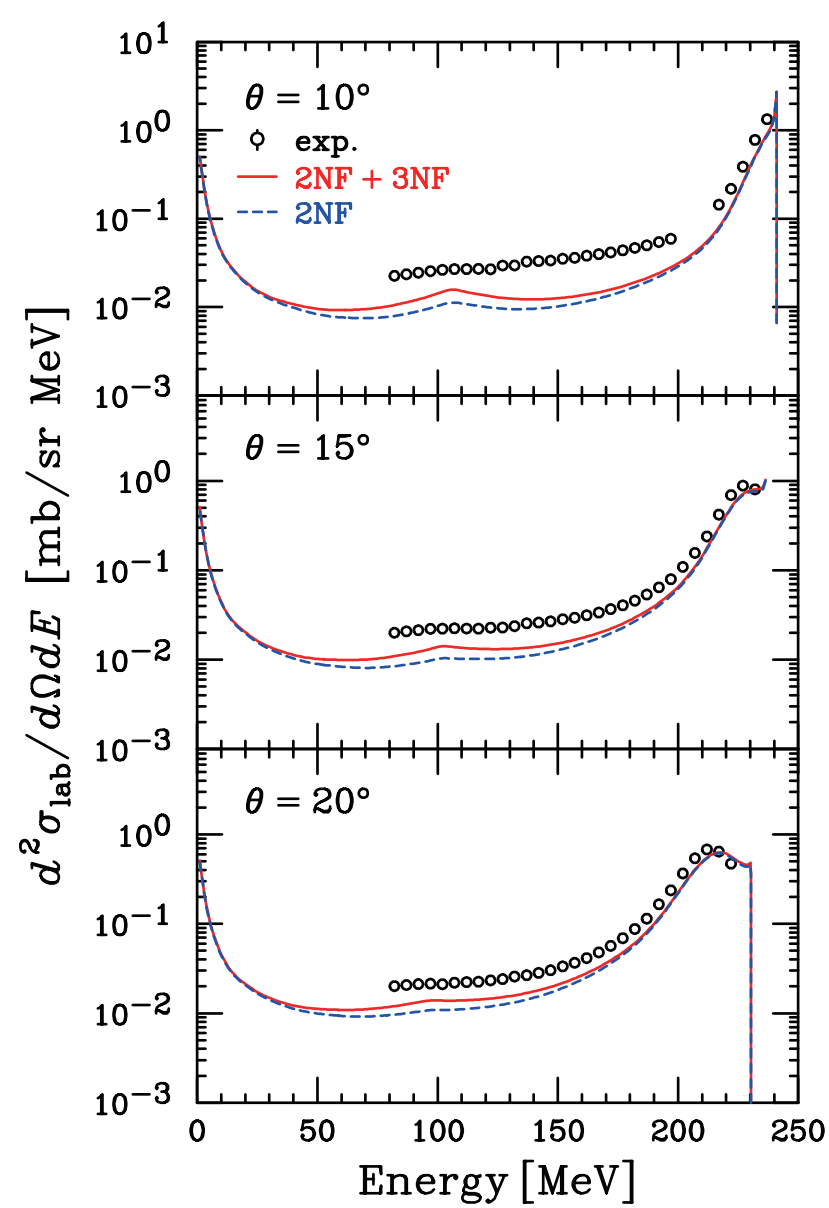

Fig. 2. Cross section of $\mathrm{D}\left(p, p_{1}\right) p_{2} n$ at $E_{p}=247 \mathrm{MeV}$. Solid and dashed curves are Faddeev calculations with and without $2 \pi 3 N \mathrm{~F}$, respectively, by Witała.

Witała. Measured cross section is larger than calculation. The disagreement increases at forward angle. Effects of $2 \pi 3 N \mathrm{~F}$ are not enough to explain the experiment. We measured also $A_{y}$ in the same experiment, but we first investigate cross section disagreement.

The disagreement is of similar magnitude with the disagreement in pd scattering cross section at background at the same energy described above. It may be natural to think that the same origin enhances both cross sections of $p d$ elastic scattering and of $p d$ breakup reaction.

In order to see microscopically the enhancement of cross section in $p d$ breakup, we recently measured $\mathrm{D}\left(p, p_{1} p_{2}\right) n$ cross section at $E_{p}=247 \mathrm{MeV}$ by detecting two protons in coincidence. We focused to investigate microscopically enhancement of $\mathrm{D}\left(p, p_{1}\right) p_{2} \mathrm{n}$ cross section at $\theta_{1}=15^{\circ}$ and $E_{1}$ being around $150 \mathrm{MeV}$. Another proton $p_{2}$ was detected at $\theta_{2}=35^{\circ}, 50^{\circ}, 65^{\circ}$ and $80^{\circ}$ on the opposite side of the beam axis, as reported in this conference by Kuroita[5]. In the same experiment, $\mathrm{D}\left(p, p_{1}\right) p_{2} n$ cross section was also measured again, and our previous data were completely confirmed.

In figure $3, \theta_{2}$ dependence of $\mathrm{D}\left(p, p_{1} p_{2}\right) n$ cross section at $E_{1}=150 \mathrm{MeV}$ is illustrated with calculations by Kamada[10]. Cross section enhancement is large at $\theta_{2}=35^{\circ}$ 
$19^{\text {th }}$ International IUPAP Conference on Few-Body Problems in Physics

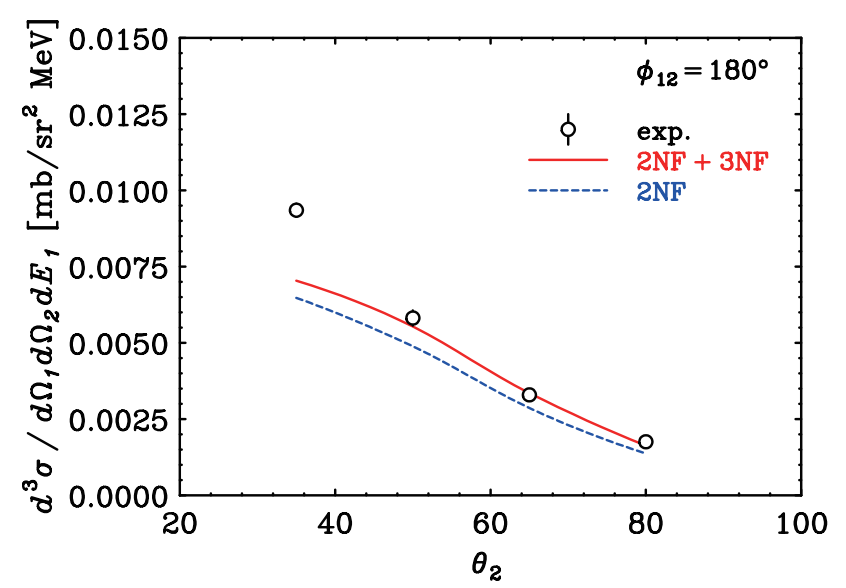

Fig. 3. $\mathrm{D}\left(p, p_{1} p_{2}\right) n$ cross section induced by $24 \mathrm{MeV} p$-beam, detected at $\theta_{1}=15^{\circ}$ and $E_{1}=150 \mathrm{MeV}$.

where cross section is large. On the contrary, cross section enhancement is small at backward $\theta_{2}$ where cross section is small. When $\theta_{1}$ and $E_{1}$ are fixed, the remaining pair of $p_{2}$ and $n$ has fixed total momentum, $\boldsymbol{p}_{\mathbf{2}}+\boldsymbol{p}_{\boldsymbol{n}}$, and absolute value of relative momentum $\left|\boldsymbol{p}_{\mathbf{2}}-\boldsymbol{p}_{\boldsymbol{n}}\right|$ is also fixed. At $\theta_{1}$ $=15^{\circ}$ and $\theta_{2}=35^{\circ}$, the three outgoing nucleons approximately form a line, i.e., satisfy the collinear condition.

Above information may be useful to guess the origin of the cross section enhancement. Experimental data at more forward angle may be also useful. To measure at forward angles we need new counters, because the present two big counters cannot come to each other closer than $48^{\circ}$ ( $=$ $\left.15^{\circ}+33^{\circ}\right)$.

\section{Experiments on $p d$ capture at higher energy}

By $p+d \rightarrow{ }^{3} \mathrm{He}+\gamma$ reaction, $p d$ scattering state comes to ${ }^{3} \mathrm{He}$ ground state. Momentum transfer is large. It is interesting to search for effects of short-range $3 \mathrm{NF}$ in this highmomentum transfer reaction. Cross section of $p d$ capture is, however, very small as below $1 \mu$ barn. Hence we used a liquid hydrogen target and detected ${ }^{3} \mathrm{He}$ recoils simultaneously in a wide angular range from $20^{\circ}$ to $160^{\circ}$ in c.m. system.

A polarized $d$-beam of energy of $196 \mathrm{MeV}$ from RCNP cyclotron was used in our first experiment. The beam polarization axis was in the vertical direction, and recoiled ${ }^{3} \mathrm{He}$ detection was made in the horizontal plane to measure $A_{y}$ and $A_{y y}$, and in the vertical plane to measure $A_{x x}$. Measured $A_{x x}$ and $A_{y y}$ took roughly the same negative values, $A_{x x} \approx A_{y y}$, and $A_{y y}$ roughly agreed with calculation, but $A_{x x}$ remarkably disagreed with calculation.

Next we measured $A_{x x}$ and $A_{y y}$ of $p d$ capture at $E_{d}$ $=137 \mathrm{MeV}$. Preliminary data indicated again the relation of $A_{x x} \approx A_{y y}$ and remarkable disagreement in $A_{x x}$. Since $p d$ capture cross section is small and identification of true events was disturbed by overwhelming background events, data analysis took time.

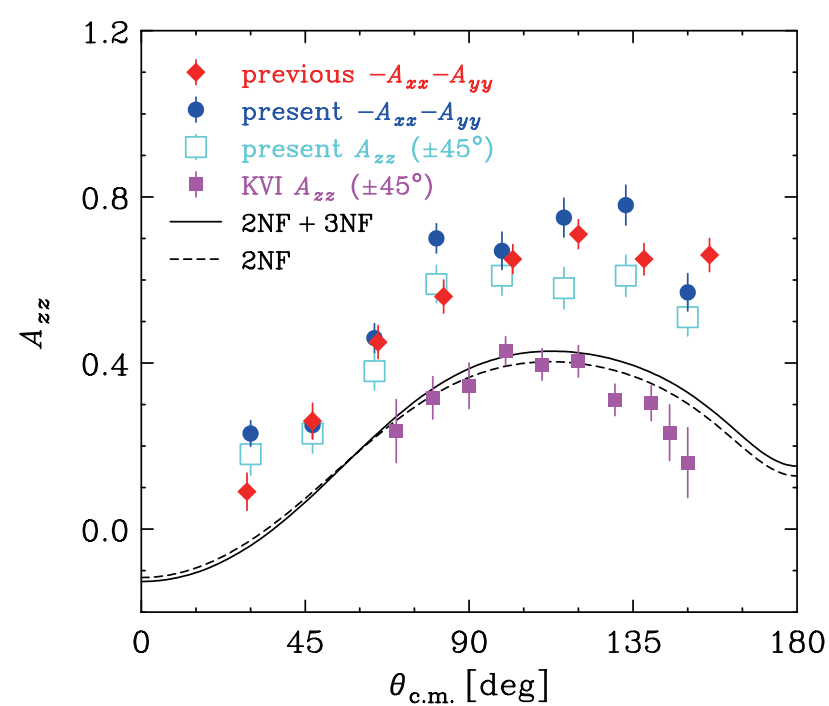

Fig. 4. " $A_{z z}$ " of $p d$ capture. Our previous data for $-A_{x x}-A_{y y}$ $\left(=A_{z z}\right)$, our new data for $-A_{x x}-A_{y y}$ and those for $A_{z z}\left( \pm 45^{\circ}\right)$ at $E_{d}=$ $196 \mathrm{MeV}$, together with calculations at $200 \mathrm{MeV}$ by Golak. KVI data for $A_{z z}\left( \pm 45^{\circ}\right)$ at $E_{d}=180 \mathrm{MeV}$ are also shown.

Meanwhile, $A_{y y}$ and $A_{z z}$ of $p d$ capture at $E_{d}=180 \mathrm{MeV}$ and $133 \mathrm{MeV}$ were measured at KVI. Their data agreed with calculations. They used a vertically polarized $d$-beam and a liquid hydrogen target, and detected ${ }^{3} \mathrm{He}$ and $\gamma$ ray in coincidence. $A_{y y}$ was measured by detecting $\gamma$-rays in the horizontal plane. $A_{z z}$ was measured by detecting $\gamma$-rays in two planes inclined by $\pm 45^{\circ}$ from the horizontal plane, and assuming the relation $A_{x x}+A_{y y}+A_{z z}=0$. KVI $A_{y y}$ data roughly agree with our data, but $A_{z z}$ data are about 1.5 times smaller in magnitude than ours.

Finally, a confirming measurement was made at RCNP on $A_{x x}, A_{y y}$ and $A_{z z}$ of $p d$ capture at $E_{d}=196 \mathrm{MeV}$. The $d$-beam was polarized in the vertical direction, ${ }^{3} \mathrm{He}$ recoils were detected in the vertical plane for $A_{x x}$, in the horizontal plane for $A_{y y}$, and in two planes inclined by $\pm 45^{\circ}$ from the horizontal plane for $A_{z z}$ in a way similar to KVI's. Dataanalysis method was improved so as to increase ${ }^{3} \mathrm{He}$ detection efficiency, and both new data and previous data were analyzed by the new method.

Our previous data and new data essentially agree to each other, and indicate the relation $A_{x x} \approx A_{y y}$ and large discrepancy in $A_{x x}$ (also in $A_{z z}$ ). Figure 4 shows various " $A_{z z}$ " data; $-A_{x x}-A_{y y}$ in our previous experiment at 196 $\mathrm{MeV},-A_{x x}-A_{y y}$ in our new experiment at $196 \mathrm{MeV}$, and $A_{z z}\left( \pm 45^{\circ}\right)$ in our new experiment at $196 \mathrm{MeV}$, together with $A_{z z}\left( \pm 45^{\circ}\right)$ in $\mathrm{KVI}$ experiment at $180 \mathrm{MeV}$. Curves are calculations at $200 \mathrm{MeV}$ with and without $2 \pi 3 N \mathrm{~F}$ by Golak. Although our " $A_{z z}$ " data are scattered to some extent, there is a large discrepancy between our " $A_{z z}$ " data and calculations.

In our data $A_{x x} \approx A_{y y}$ relation holds, but calculated $A_{x x}$ and $A_{y y}$ are significantly different to each other, therefore a large discrepancy in $A_{x x}$ (also in $A_{z z}$ ) results. The relation $A_{x x} \approx A_{y y}$ means the symmetry of $p d$ capture with respect to the $z$-axis (the beam axis). When a $d$-beam is polarized in $y$-direction (vertically), $d$-induced reactions in the verti- 
cal (yz) plane and in the horizontal $(x z)$ plane are expected to proceed differently in general. Therefore it is natural to expect $A_{x x} \neq A_{y y}$ also in the present case.

In many other $d$-induced reactions, a relation $A_{x x} \approx$ $-A_{y y}$ holds approximately. A deuteron has a prolate shape. If a $d$-beam is vertically polarized and $d$-induced reactions take place in peripheral region of target nuclei, the reactions may be enhanced in vertical plane and suppressed in horizontal plane, or oppositely suppressed and enhanced.

The relation $A_{x x} \approx A_{y y}$ in $p d$ capture is curious. It is interesting to investigate the origin(s) of this relation, including to estimate effects from short-range $3 \mathrm{NF}$.

\section{Experiments on $p d$ star anomaly at low energy}

Discrepancies at higher energy have candidates for their origin(s), e.g., short-range $3 N \mathrm{~F}$, relativity, high-angular momentum reactions. On the contrary, discrepancies at lower energy have not apparent candidates for their ori$\operatorname{gin}(\mathrm{s})$.

$A_{y}$ puzzle is well known for a long time. We have already enough data sets for $A_{y}$ puzzle and no systematic measurements on $A_{y}$ puzzle have been made recently. Many theoretical attempts such as modifications of $2 \mathrm{NF}$ and introduction of $L S$ dependent $3 N \mathrm{~F}$ have been examined, but $A_{y}$ puzzle has not been solved yet.

Another big problem at low energy is Space-Star anomaly. SS anomaly was found first in $n d$ breakup at $E_{n}=$ $13 \mathrm{MeV}$ and $10.5 \mathrm{MeV}$ [11], and was confirmed at $13 \mathrm{MeV}$ by another experiment [12]. Experiments of $p d$ breakup at SS configuration were also made, and SS anomaly in $p d$ breakup was first found when a reliable $p d$ calculation was made [6].

At $13 \mathrm{MeV}$, nd breakup cross section at SS configuration is about $25 \%$ higher than calculation, and $p d$ breakup cross section at SS is about $15 \%$ lower than calculation. So far no theoretical suggestions have been made for SS anomaly and its large charge asymmetry.

Because a reliable calculation on $p d$ breakup has become available since 2005 [6], we have been making a systematic measurement of $p d$ star cross section at $E_{p}=$ $13 \mathrm{MeV}$ and 9.5 MeV. At Koeln University, measurement of $p d$ star cross section at $E_{d}=19 \mathrm{MeV}\left(E_{p}=9.5 \mathrm{MeV}\right)$ was made[13].

When three outgoing nucleons from $N d$ breakup have the same energy and form an equilateral triangle, we call the configuration as Star. When the Star triangle is perpendicular to the beam axis, we call the configuration as Space Star. An angle between Star plane and the beam axis in c.m. frame is called as $\alpha$, as defined in Fig. 5. We usually detect two protons from $p d$ breakup at symmetrical angles with respect to the beam axis, and we define $\alpha=0^{\circ}$ when a $p$-beam is used and two detected protons are at forward angles in the horizontal plane. See Figure 5.

To see characteristics of Star anomaly, $\alpha$-dependence of $p d$ Star anomaly has been measured recently by Koeln group[13] and Kyushu group. Star configuration at $\alpha=0^{\circ}$

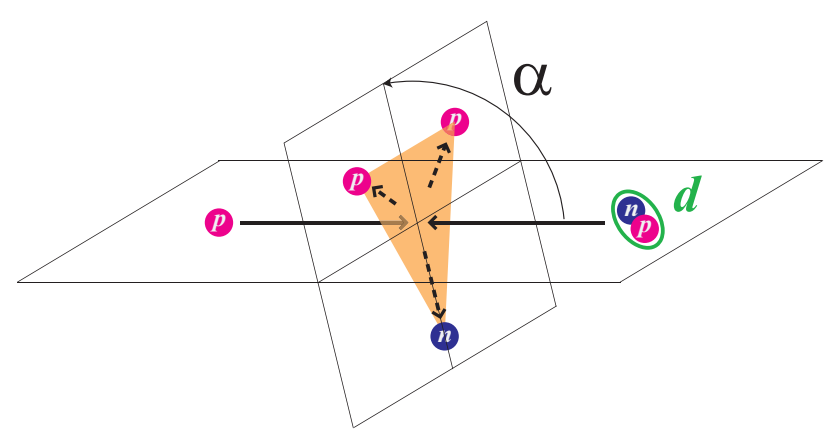

Fig. 5. Definition of an inclination angle $\alpha$ for Star configuration.

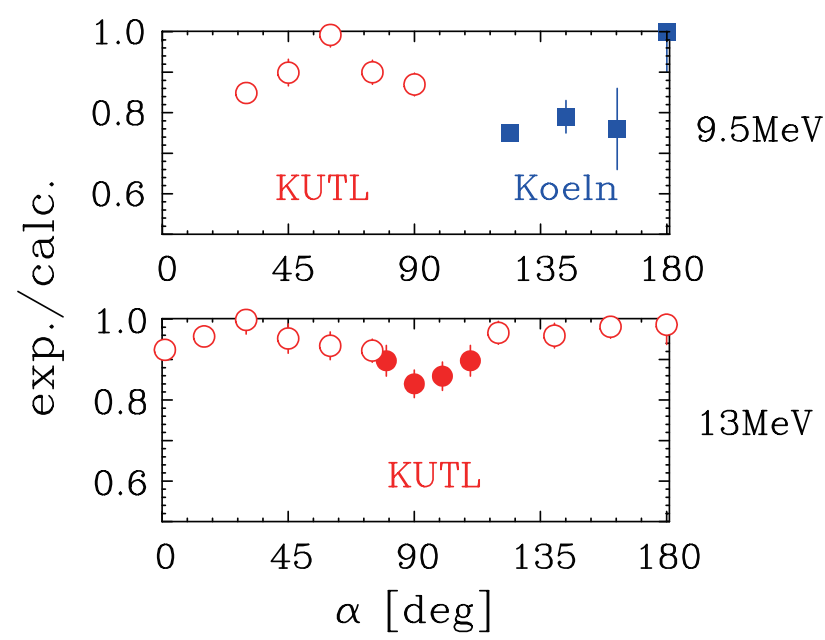

Fig. 6. Recent results for $\alpha$-dependence of $p d$ Star anomaly at $E_{p}$ $=9.5 \mathrm{MeV}\left(E_{d}=19 \mathrm{MeV}\right)$ and at $E_{p}=13 \mathrm{MeV}\left(E_{d}=26 \mathrm{MeV}\right)$.

is close to QFS configuration, and possible anomaly at QFS is also being investigated at Kyushu. Y. Maeda and Y. Eguchi report on these subjects in this conference.

As seen in Figure 6, star anomaly at $13 \mathrm{MeV}$ is confined at around $90^{\circ}$. The plane perpendicular to the beam axis is special. Only in the perpendicular plane, $p d$ breakup reaction is suppressed. It seems to be enough to think of curious suppression in the perpendicular plane.

At $9.5 \mathrm{MeV}$, however, remarkable Star anomaly appears also at backward angles, as indicated by Koeln experiment at $E_{d}=19 \mathrm{MeV}$. Complex consideration may be necessary to explain the wide-range Star anomaly.

Before thinking of origins of $p d$ Star anomaly, it is better to make a confirming experiment at $E_{d}=19 \mathrm{MeV}$. A polarized $d$-beam was used in Koeln experiment, but an unpolarized $d$-beam will be used in the confirming experiment to measure cross section alone.

Our strategy is (a) confirmation of $p d$ Star anomaly by additional experiments, (b) investigation of origin(s) of $p d$ Star anomaly, then (c) elucidation of $n d$ Star anomaly. So far large charge asymmetry between $n d$ SS anomaly and $p d$ SS anomaly has been reported. The large charge asymmetry is hard to explain. We will first elucidate $p d$ Star anomaly, based on systematic and reliable measurements. Elucidation of $p d$ Star anomaly may include suggestion on the charge asymmetry. Experimental data for $n d$ Star are insufficient at present, and reliable $n d$ experiments are hard 
to make. So we will not investigate $n d$ Star anomaly till $p d$ Star anomaly is completely elucidated.

\section{Experiments on $p d$ QFS at low energy}

Cross section enhancement of 16-18\% was reported in $n d$ QFS at $E_{n}=25 \mathrm{MeV}$ and $26 \mathrm{MeV}$. Also cross section suppression in $p d$ QFS was reported at $E_{p}=10.5 \mathrm{MeV}$ and 19 $\mathrm{MeV}$.

We are making systematic measurement of $p d$ QFS cross section at KUTL, and no apparent $p d$ QFS anomaly has been found at both $9.5 \mathrm{MeV}$ and $13 \mathrm{MeV}$. We will measure $p d$ QFS cross section at $10.5 \mathrm{MeV}$ and $19 \mathrm{MeV}$ to see if $p d$ QFS anomaly exists or not.

\section{Summary}

Studies of $3 N$ systems are summarized and illustrated in Figure 1 . We are on the way to search for short-range $3 N \mathrm{~F}$ at higher energy in $p d$ scattering, $p d$ breakup and $p d$ capture, and to investigate origins of Star anomaly as well as of $A_{y}$ puzzle at low energy. Experimental studies have made steady progress. At low energy, success of reliable $p d$ calculation enabled systematic studies of $p d$ Star anomaly.

Challenging $3 N$ calculations aiming to solve remaining problems in $3 N$ reactions are expected.

\section{Acknowledgement}

The author thanks collaborators of many experiments in $p d$ capture, $p d$ breakup and $p d$ Star. The collaborators are from Kyushu University, RCNP Osaka University, RIKEN, CNS University of Tokyo and Tohoku University at the time of experiment. The author also appreciate collaboration of theorists, H. Kamada, H. Witała, J. Golak and A. Deltuva for their calculations and discussions. Owing to their efforts, we have made great progress in investigation of problems in $3 N$ reactions in the last decade. The next decade will be fruitful.

\section{References}

1. J. Fujita and H. Miyazawa, Prog. Theor. Phys. 17, (1957) 360.

2. K. Sagara et al., Phys. Rev. C50, (1994) 576.

3. Y. Koike and S. Ishikawa, Nucl. Phys. A631, (1998) 683c.

4. H. Witała et al., Phys. Rev. Lett. 81, (1998) 1183.

5. S. Kuroita, Proceedings of FB19 (2010).

6. A. Deltuva et al., Phys. Rev. C72, (2005) 054004.

7. K. Hatanaka et al., Phys. Rev. C66, (2002) 044002.

8. K. Sekiguchi et al., Phys. Rev. C65, (2002) 034003.

9. H. Witała, private communication.

10. H. Kamada, private communication.

11. J. Strate et al., Nucl. Phys. A501, (1989) 51.

12. H.R. Setze et al., Phys. Rev. C75, (2005) 034006.

13. J. Ley et al., Phys. Rev. C75, (2006) 064001. 